\title{
Illumina-Based Analysis of Endophytic and Rhizosphere Bacterial Diversity of the Coastal Halophyte Messerschmidia sibirica
}

\author{
Xue-Ying Tian and Cheng-Sheng Zhang* \\ Marine Agriculture Research Center, Tobacco Research Institute of Chinese Academy of Agricultural Sciences, Qingdao, \\ China
}

Halophytes play important roles in coastal ecosystems. However, few reports have described bacterial communities related to halophytes, and the distribution patterns of these bacteria in different plant tissues have been rarely compared. This paper mainly studied the diversity and community structure of endophytic and rhizosphere (Rh) bacteria related to the halophyte Messerschmidia sibirica, a dominant species in the coastal zone of Shandong Peninsula, China. We collected leaf (Lf), stem (Sm), root (Rt), $\mathrm{Rh}$, and bulk (Bl) control soil samples, and sequenced the V5-V7 region of the bacterial

OPEN ACCESS

Edited by:

Paula García-Fraile, Academy of Sciences of the Czech

Republic (ASCR), Czechia

Reviewed by:

Devin Coleman-Derr

University of California, Berkeley,

United States

Lorena Carro,

University of Newcastle, Australia

*Correspondence:

Cheng-Sheng Zhang

zhchengsheng@126.com

Specialty section

This article was submitted to

Microbial Symbioses,

a section of the journal

Frontiers in Microbiology

Received: 11 June 2017 Accepted: 06 November 2017

Published: 20 November 2017

Citation:

Tian X-Y and Zhang C-S (2017) Illumina-Based Analysis of Endophytic and Rhizosphere Bacterial Diversity of the Coastal Halophyte Messerschmidia sibirica.

Front. Microbiol. 8:2288.

doi: 10.3389/fmicb.2017.02288
16S rRNA gene using the Illumina HiSeq platform to identify bacterial communities originating from different plant habitats. We found that the bacterial richness and diversity in Rh were significantly higher than those in the leaves, Sm, and Rt, but lower than those of the Bl control soil. In total, 37 phyla and 438 genera were identified. Microbial-diversity analysis showed that Proteobacteria and Actinobacteria were the dominant phyla and that Pseudomonas, Bacillus, Sphingomonas, Streptomyces, Microbacterium, Rhizobium, and Nocardioides were the dominant genera. However, there were clear differences in community diversity and structure among the samples. Endophytic bacteria community in Lf, Sm, and Rt shared more similarity than those in $\mathrm{Rh}$ and $\mathrm{Bl}$ control soil. The numbers of operational taxonomic units exclusive to the Lf, stem, Rt, Rh, and Bl control soil samples were 51, 43, 122, 139, and 922, respectively, implying habitat-specific patterns. Principal coordinate analysis demonstrated differences were apparent in the bacterial communities associated with habitats. On the whole, M. sibirica affected bacterial diversity and structured the bacterial community. This study provides insight into the complex microbial compositions of coastal halophytes.

Keywords: Messerschmidia sibirica, halophyte, endophytic bacteria, rhizosphere bacteria, diversity, Illumina HiSeq

\section{INTRODUCTION}

Along with emerging research on plant-microbe interactions, accumulating evidence suggests that endophytic and rhizosphere (Rh) bacteria play important roles in plant growth. These microbes can benefit plants by producing plant hormones (Etesami et al., 2015), enabling nutrient uptake (Silveira et al., 2016), increasing stress resistance (Yi et al., 2016; Shahzad et al., 2017), or altering the 
Rh microbial balance (Laanbroek et al., 2013; Chen et al., 2017). Moreover, the bacterial community and diversity vary across different plant species (Ramírez-Elías et al., 2014), colonization sites (Jin et al., 2014), and growing environments (Xu et al., 2014). Therefore, research into endophytic and Rh bacterial diversity is important for clarifying the functions of these bacteria and excavating these bio-resources. In recent years, most studies have focused on microbes associated with glycophytes or crop species (Xu et al., 2014; Haque et al., 2015; Majeed et al., 2015), while reports on bacterial communities associated with plants in extreme environments are limited.

The intertidal zone is an important part of the coastal environment, exhibiting characteristics of both marine and terrestrial ecosystems (Wiegert et al., 1981). Because of the characteristics of coastal environments (such as high salinity and tidal changes), a large number of halophytes grow there and perform important ecological functions to maintain the balance of the intertidal ecosystem. Halophytes have evolved various strategies to survive in this saline environment, and bacteria associated with halophytic plants may be key factors in such strategies. A large number of endophytic bacteria from halophytes have been shown the ability to improve salt tolerance of their host plants. For example, NavarroTorre et al. (2017) reported that the endophytic bacteria from Arthrocnemum macrostachyum could improve its salt tolerance. Hashem et al. (2016) revealed that the growth promotion effect of endophytic bacteria and arbuscular mycorrhizal fungi (AMF) on Acacia gerrardii under salt stress were associated to a tripartite mutualistic symbiosis in A. gerrardii. Moreover, these microorganisms have been expected to be used in microbialassisted phytoremediation of saline soils (Syranidou et al., 2016; Zhao et al., 2016). Different plant organs and the $\mathrm{Rh}$ may influence the abundances of bacteria and structures of bacterial communities, and it is therefore important to understand these parameters.

Messerschmidia sibirica is a salt-secreting halophyte that is widely distributed on the sandy beaches of northern coastal areas in China (Xiang et al., 2008). M. sibirica is a useful germplasm resource with high commercial value and ecological importance. For example, it can be used for environmental protection, such as sand stabilization, soil improvement, and phytoremediation (Jin et al., 2012). M. sibirica also has value in the medical, feed, and landscaping industries (Wang and Zheng, 2016). Consequently, research into the diversity of endophytic and $\mathrm{Rh}$ bacteria related to $M$. sibirica makes a great contribution to understand the connection between these microbes and the plant's tolerance to high salt and infertile soil environments. They can also be potentially used in agricultural, biotechnological, and medical fields. It has been confirmed that endophytic bacteria from M. sibirica exhibited good plant growth-promoting ability (Shin et al., 2007). However, little information is available about the endophytic and Rh bacterial community of the plant. Thus, the objective of the present study was to obtain a broad overview of the bacterial community structures of different tissues and the $\mathrm{Rh}$ of $M$. sibirica, as well as the surrounding bare soil, using high-throughput sequencing. This represents the first study to characterize endophytic and $\mathrm{Rh}$ bacteria related to the halophyte

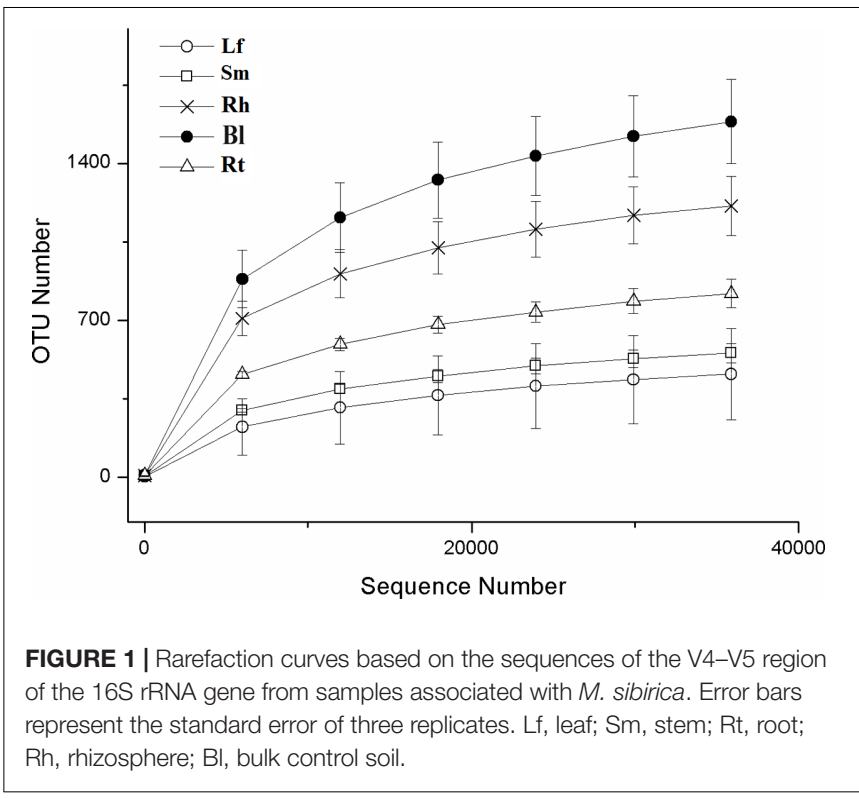

M. sibirica in a Chinese coastal area. Our results provide new insight into this bacterial community and a foundation for future studies.

\section{MATERIALS AND METHODS}

\section{Sample Collection and Surface Sterilization}

Sampling sites located in the Qingdao coastal zone $\left(36^{\circ} 5^{\prime} 23^{\prime \prime} \mathrm{S}\right.$ and $120^{\circ} 27^{\prime} 55^{\prime \prime} \mathrm{E}$ ) in the Shandong Province of China. The local area has a temperate continental climate, possessing a mean air temperature of $13^{\circ} \mathrm{C}$ and an annual rainfall of 530-630 mm (Ma et al., 2013). M. sibirica is the dominant species in the intertidal zone. Three sampling sites of approximately $500 \mathrm{~m}$ each were selected, and three healthy plants were randomly collected from each site in July 2016. Sterilized gloves and spades were used to collect the samples, and the spades were sterilized between each collection. The mixture obtained from the three plants at the same sampling site constituted a sample. Samples collected included leaves (Lf), stems (Sm), roots (Rt), Rh soil, and bulk (Bl) control soil. The soil close to the Rt $(0-3 \mathrm{~mm}$ from the Rt surface) was defined as the Rh soil (Zhang and Kong, 2014). Samples collected from locations $10-15 \mathrm{~cm}$ away from the Rt were considered as $\mathrm{Bl}$ control soil samples (Smalla et al., 2001). All samples were placed in aseptic bags which placed ice straightway and transported back to our lab. The plant materials (Lf, Sm, and Rt) were surface-sterilized according to Pereira and Castro (2014) method.

\section{DNA Extraction, PCR Amplification, and Gene Clone Library Construction}

After the addition of liquid nitrogen, Lf, Sm, and Rt samples were frozen and ground rapidly to a fine powder in a sterilized and pre-cooled mortar, then transferred to a bead tube. For the Rh 
TABLE 1 | Operational taxonomic unit (OTU) richness and diversity indices of different samples associated with $M$. sibirica with a $97 \%$ similarity cut-off.

\begin{tabular}{|c|c|c|c|c|c|c|}
\hline Sample name & Sample origin & OTUs observed & Shannon & Chao1 & ACE & Coverage (\%) \\
\hline Lf & Leaf & $527 \pm 74 d$ & $4.02 \pm 0.65 d$ & $465 \pm 93 d$ & $480 \pm 92 d$ & 99.7 \\
\hline Sm & Stem & $553 \pm 42 d$ & $5.12 \pm 0.29 c$ & $671 \pm 66 \mathrm{~cd}$ & $666 \pm 59 d$ & 99.6 \\
\hline Rt & Root & $821 \pm 63 c$ & $6.64 \pm 0.19 b$ & $979 \pm 168 c$ & $990 \pm 144 \mathrm{c}$ & 99.5 \\
\hline $\mathrm{Rh}$ & Rhizosphere & $1210 \pm 188 b$ & $7.90 \pm 0.48 a$ & $1351 \pm 148 b$ & $1375 \pm 144 b$ & 99.4 \\
\hline $\mathrm{Bl}$ & Bulk control soil & $1587 \pm 132 \mathrm{a}$ & $8.35 \pm 0.61 \mathrm{a}$ & $1847 \pm 234 a$ & $1853 \pm 220 a$ & 99.0 \\
\hline
\end{tabular}

Values are the means of three replicates $\pm S D$. Values within a column followed by different lowercase letters are significantly different (P < 0.05$)$.

and $\mathrm{Bl}$ samples, $0.5 \mathrm{~g}$ of each sample was used for DNA extraction. Total DNA extraction was performed based on the instructions of the PowerSoil ${ }^{\circledast}$ DNA Isolation Kit. DNA was stored at $-20^{\circ} \mathrm{C}$ until subsequent analysis.

The target-specific primers 799F (5'-AACMGGATTAG ATACCCKG-3') and 1193R (5'-ACGTCATCCCCACCTT CC- $\left.3^{\prime}\right)$, which do not amplify the chloroplast or mitochondrial $16 \mathrm{~S}$ rRNA genes of $M$. sibirica, were used to amplify the V5-V7 region of the bacterial 16S rRNA gene (Beckers et al., 2016). Every PCR reaction was performed with Phusion ${ }^{\circledR}$ High-Fidelity PCR Master Mix (New England BioLabs, Ipswich, MA, United States). Finally, sequencing and construction of the 16S rRNA gene clone libraries were performed at Novogene (Beijing, China) using the Illumina HiSeq 2500 platform, and 250-bp paired-end reads were created.

\section{Sequence Processing and Analysis}

Paired-end reads were merged using FLASH software (v1.2.7; Magoč and Salzberg, 2011). Using QIIME (v1.7.0; Bais et al., 2006) processing and the UCHIME arithmetic (Edgar et al., 2011), and then obtained effective tags. Each operational taxonomic unit (OTU) was defined as a cluster of reads with $97 \%$ sequence identity. Each OTU was annotated with the SSU-rRNA SILVA database ${ }^{1}$ using MOTHUR (Wang et al., 2007). The complete sequences generated in this study are available in the NCBI SRA database under accession number SRR6161354 (Lf), SRR6161411 (Sm), SRR6161618 (Rt), SRR6161623 (Rh), and SRR6161637 (Bl).

The dataset without singletons was rarefied to the lowest number of reads $(35,880)$ recovered from our samples for comparative analysis of species richness and diversity indices (Chao1, Shannon, Simpson, ACE, and good-coverage) among samples, using QIIME (v1.7.0; Kuczynski et al., 2011).

\section{Statistical Analysis}

Statistical analyses were performed with the vegan package (Oksanen et al., 2011) in R (v2.15.3). One-way ANOVAs and Tukey-Kramer tests were used to test for differences in sample alpha diversities (observed OTUs and Shannon, Chao 1, and ACE richness and diversity indices). Heatmap images were created using the R package "heatmap" program (Kolde, 2012), and Venn diagrams were produced with the Venn-Diagram program (Chen, 2012). To explore variation in bacterial community compositions among samples, correspondence analysis (CA)

${ }^{1}$ http://www.arb-silva.de/

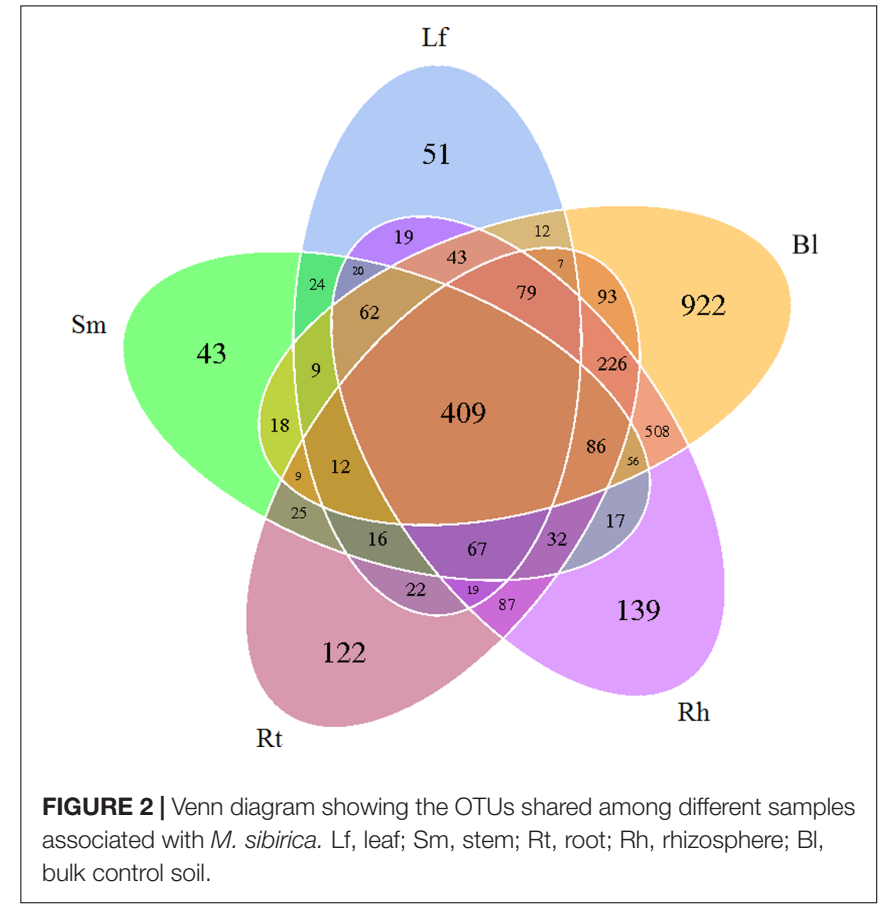

was conducted in the $\mathrm{R}$ (v2.15.3) package ca (Nenadic and Greenacre, 2007). The relationships between bacterial community structures were evaluated by principal coordinate analysis (PCoA). Furthermore, we use LEfSe software (v1.0) to identify differentially abundant families among samples for biomarker discovery (Segata et al., 2011). Significant distinctions in the constituent of bacterial communities from the various habitats were determined with Adonis.

\section{RESULTS}

\section{Characteristics of Sample Sequence Tags}

After read-quality filtering, a total of 821,613 high-quality sequences were queried. The number of high-quality reads per sample ranged from 46,290 to 68,650 (average length of 376-378 bp) (Supplementary Table S1). Rarefaction curves (Figure 1), combined with the estimated coverage values (Table 1), suggested that the libraries were sufficiently large to capture a large majority of the bacterial diversity in the samples used in this study. Interestingly, the rarefaction curves indicated 


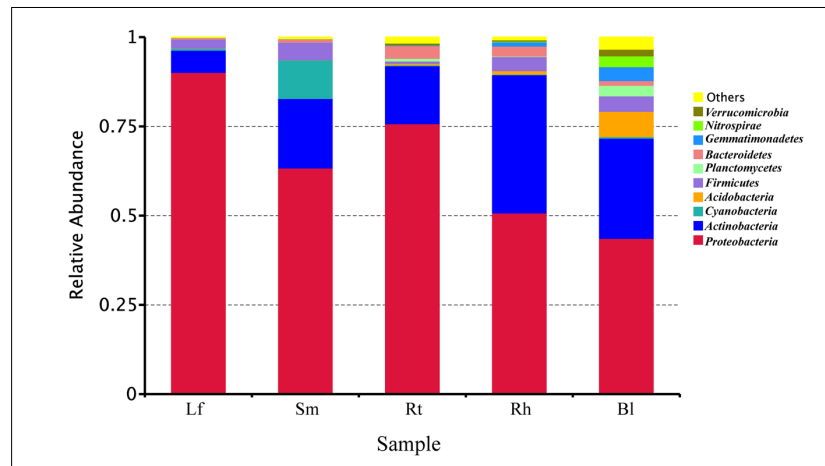

FIGURE 3 | Relative abundances of bacteria at the phylum level in different samples. Lf, leaf; Sm, stem; Rt, root; Rh, rhizosphere; BI, bulk control soil.

higher OTU numbers in the Rt samples than in the Lf and Sm samples, as well as higher numbers in the $\mathrm{Bl}$ control soil samples than in the Rh samples. A total of 3254 OTUs were detected across all libraries with 403 OTUs common to all samples (Figure 2). The numbers of OTUs exclusive to the Lf, $\mathrm{Sm}$, $\mathrm{Rt}, \mathrm{Rh}$, and $\mathrm{Bl}$ samples were $51,43,122,139$, and 922, respectively. The shared bacterial OTUs mainly belonged to the Proteobacteria (229), Actinobacteria (127), Firmicutes (28), and Bacteroidetes (10) at the phylum level and Rhizobium (21), Bacillus (17), Sphingomonas (15), and Streptomyces (11) at the genus level.

\section{Microbial Community Richness and Diversity}

The richness and diversity values of the bacterial communities after normalization are shown in Table $\mathbf{1}$ and Figure 1. Bl samples harbored the highest number of OTUs. The Chaol and ACE richness estimators and Shannon index were calculated based on

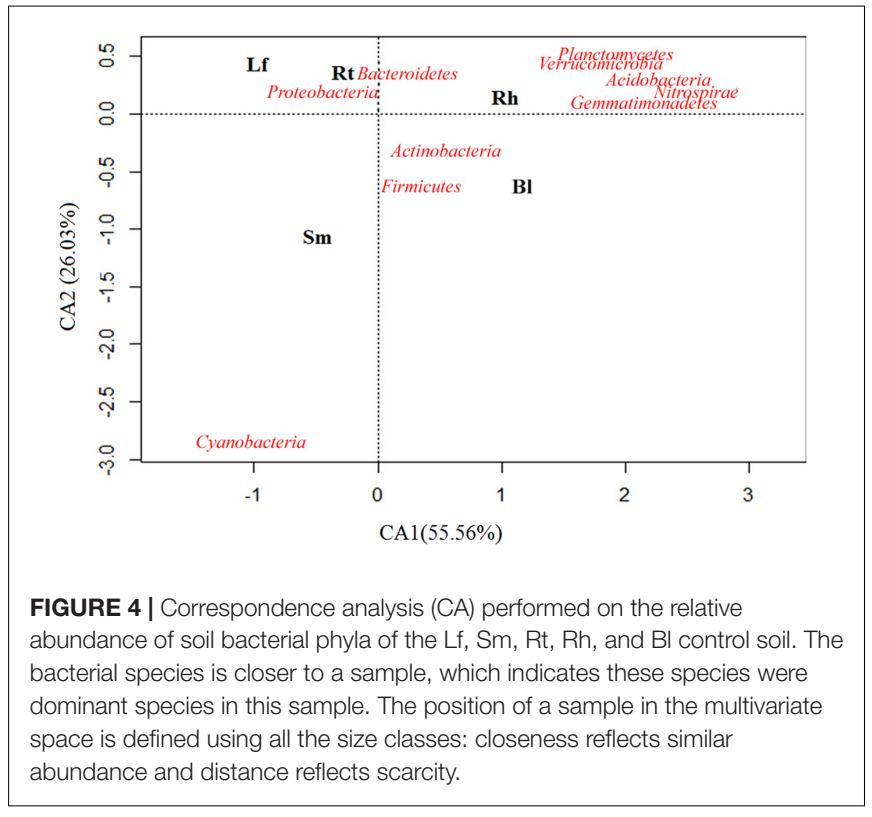

a 3\% genetic distance for all samples (Table 1), and all values were highest in the Bl samples, followed by Rh, Rt, Lf, and Sm.

\section{Microbial Taxonomic Analysis at the Phylum Level}

High-throughput sequencing revealed the diversity of bacterial communities in different samples at the phylum level (Figure 3). Thirty-seven phyla were identified, and the relative abundances of the top 10 phyla (each relative abundance, $>1 \%$ ) are shown in Figure 3. The phyla Proteobacteria, Actinobacteria, Cyanobacteria, Acidobacteria, Firmicutes, Planctomycetes, Bacteroidetes, Gemmatimonadetes, Nitrospirae, and Verrucomicrobia were detected in all samples; however, their relative abundances varied across different samples. Proteobacteria and Actinobacteria were the predominant bacterial phyla, accounting for more than 43.7 and $6.2 \%$, respectively, of all samples. The abundance of Cyanobacteria was $10.8 \%$ in the Sm samples, which was much higher than that in the other samples. Acidobacteria, Planctomycetes, Nitrospirae, and Verrucomicrobia were higher in the $\mathrm{Bl}$ samples, while Firmicutes were lower in the Rt than in other samples.

Samples were scattered among the four quadrants in the CA analysis plot (Figure 4), suggesting the presence of different dominant bacterial communities in different samples, although the major bacterial communities appeared similar between the Lf and Rt samples. In addition, communities in the Rt and Rh samples were relatively similar compared to those in other organs or habitats, and this was due to the assignment of more sequences to the Bacteroidetes and Proteobacteria phyla. Cyanobacteria appeared along the edge of the graph, indicating that this phylum may have appeared sporadically or in a special habitat. Although CA provides only a broad resolution, it indicated a trend for different bacterial compositions in different habitats. For example, the enrichment of Acidobacteria in the $\mathrm{Rh}$ and $\mathrm{Bl}$ samples indicates that they appear to live in the $\mathrm{Rh}$ and $\mathrm{Bl}$ soils.

\section{Microbial Taxonomic Analysis at the Genus Level}

Clustering of the top 35 genera is shown in Figure 5 (supporting data are shown in Supplementary Table S2). These classified bacterial genera belonged to five phyla. Among these, 19 genera belonged to Proteobacteria, 13 to Actinobacteria, 1 to Cyanobacteria, 1 to Firmicutes, and 1 to Bacteroidetes. The following genera were the most abundant $(>0.2 \%)$ across all samples: Pseudomonas, Bacillus, Sphingomonas, Streptomyces, Microbacterium, Rhizobium, and Nocardioides. However, species distributions differed greatly across different samples. Methylobacterium, Aureimonas, Sphingomonas, Providencia, and Pseudomonas were mainly distributed in the Lf, while Bacillus, Derratia, Kocuria, Massilia, and Curtobacterium were dominant in the Sm. Similarly, Rt samples possessed more species belonging to Rhizobium, Novosphingobium, Sphingopyxis, Hydrogenophaga, Herbiconiux, Streptomyces, Hyphomicrobium, Steroidobacter, Bradyrhizobium, and Variibacter. Dominant genera in the Rh included Paracocccus, Aeromicrobium, Devosia, 


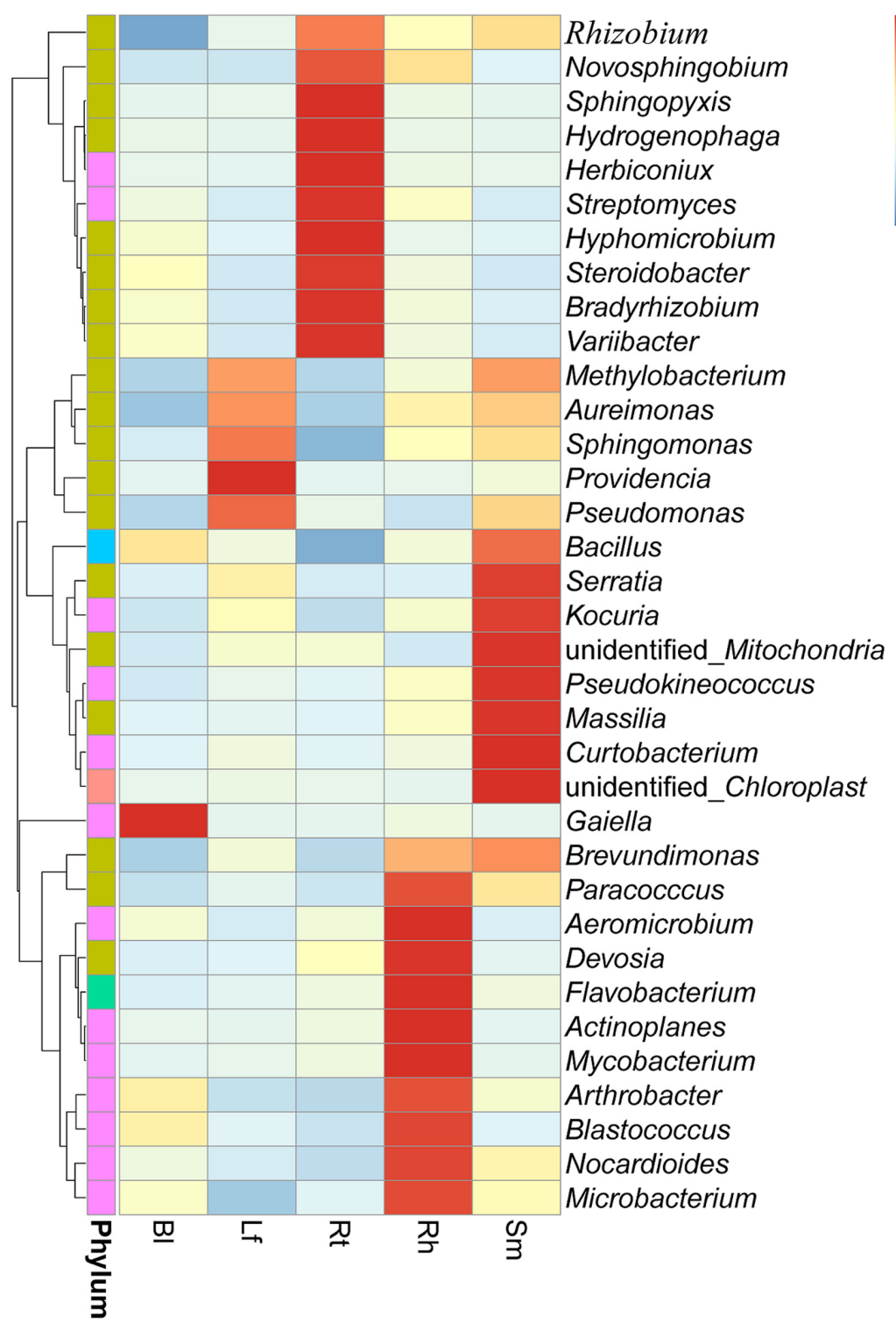

\subsection{Phylum \\ $1 \quad$ Actinobacteria \\ $0.5 \quad$ Bacteroidetes \\ $-0.5 \quad$ Cyanobacteria \\ -1 Firmicutes \\ Proteobacteria}

FIGURE 5 | Heatmap displaying the relative abundances of the most dominant genera (top 35) in each sample. The dendrogram represents complete-linkage agglomerative clustering, based on Euclidean dissimilarities. Lf, leaf; Sm, stem; Rt, root; Rh, rhizosphere; Bl, bulk control soil.

Flavobacterium, Actinoplanes, Mycobacterium, Arthrobacter, Blastococcus, Nocardioides, and Microbacterium. Only one genus, Gaiella, was predominantly distributed in the $\mathrm{Bl}$ control soil. Pseudomonas was the dominant genus in Lf (25.6\%) and Sm (17.65\%) samples, while Rhizobium, Actinoplanes, and Gaiella were dominant in Rt (10.07\%), Rh (5.79\%), and $\mathrm{Bl}(3.71 \%)$ samples, respectively. The relative abundance of Pseudomonas was higher in Lf samples (25.6\%) than in the other samples.

\section{Comparative Analysis of Bacteria in Different Sample Groups}

Important distinctions were found in the compositions of bacterial communities in the five sample groups.
Significantly different taxa abundances were found among the different samples, as determined by LEfSe (Figure 6). At the family level, Pseudomonadaceae, Enterobacteriaceae, and Aurantimonadaceae were significantly enriched in the Lf samples, while Kineosporicaceae and Oxalobacteraceae were more abundant in the Sm samples. Bradyrhizobiaceae, Xanthobacteraceae, and Comamonadaceae exhibited relatively higher abundances in the Rt samples. Geodermatophilaceae, Nocardioidaceae, and Hyphomicrobiaceae were abundant in the $\mathrm{Rh}$ samples. Four families showed significantly different abundances across samples: Gaiellaceae, Gemmatimonadaceae, Nitrospiraceae, and Nitrosomonadaceae. These differentially abundant taxa can be considered as potential biomarkers $($ LDA $>2, P<0.05)$. 

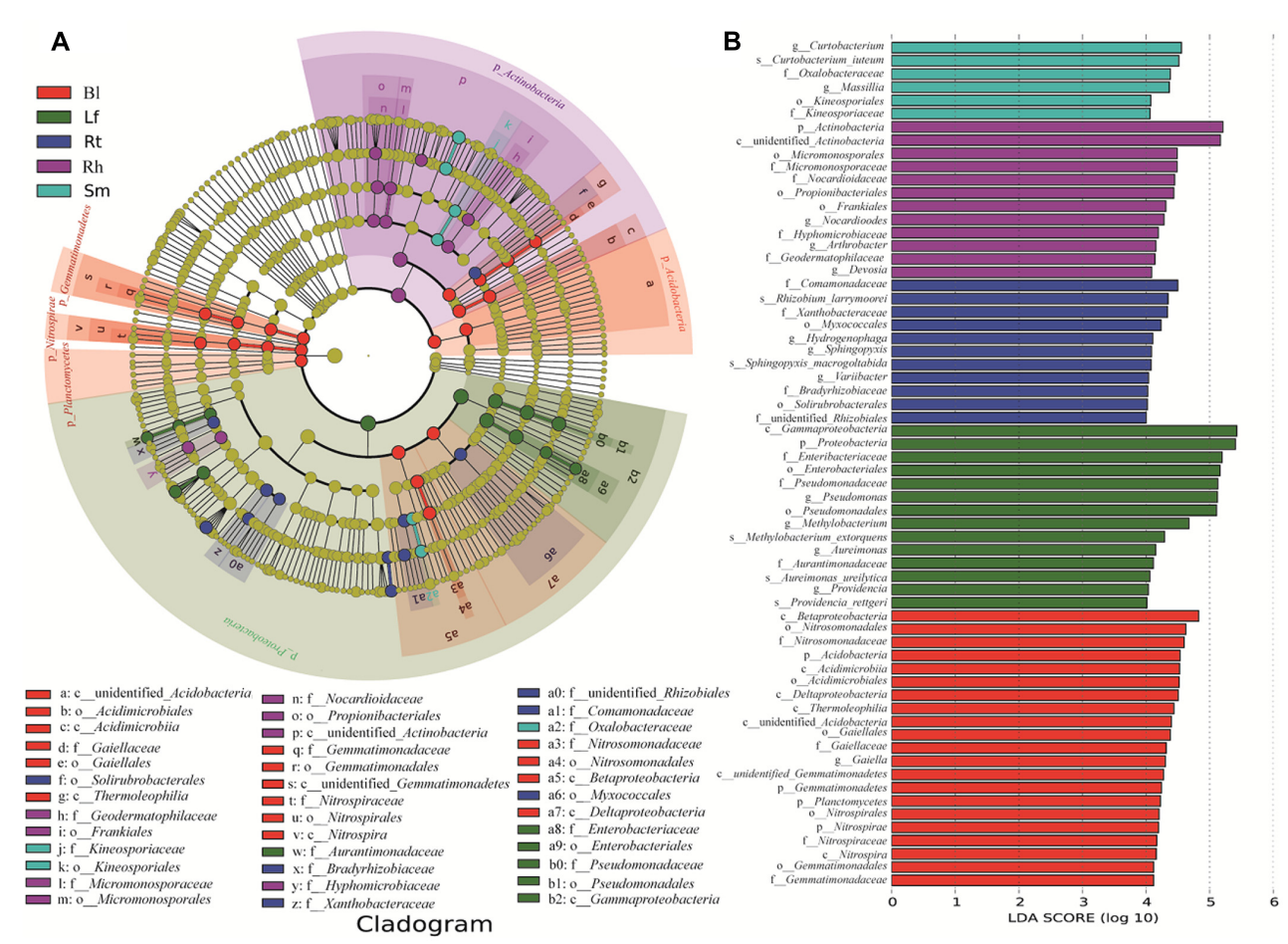

FIGURE 6 | Groups from the phylum-to-family level determined to be significant representatives of their sample type, based on LefSe software analysis.

(A) Cladogram representing the taxonomic hierarchical structure of the identified habitat biomarkers generated using LEfSe is shown. Each ring represents a taxonomic level, with phylum, class, order, and family emanating from the center to the periphery. Each circle is a taxonomic unit found in the dataset, with circles or nodes shown in color where the taxon represented a significantly more abundant group. (B) Identified biomarkers ranked by the effect size in different samples. The habitat biomarkers were identified as being significantly abundant (alpha value < 0.05) when compared among samples. Lf, leaf; Sm, stem; Rt, root; Rh, rhizosphere; Bl, bulk control soil.

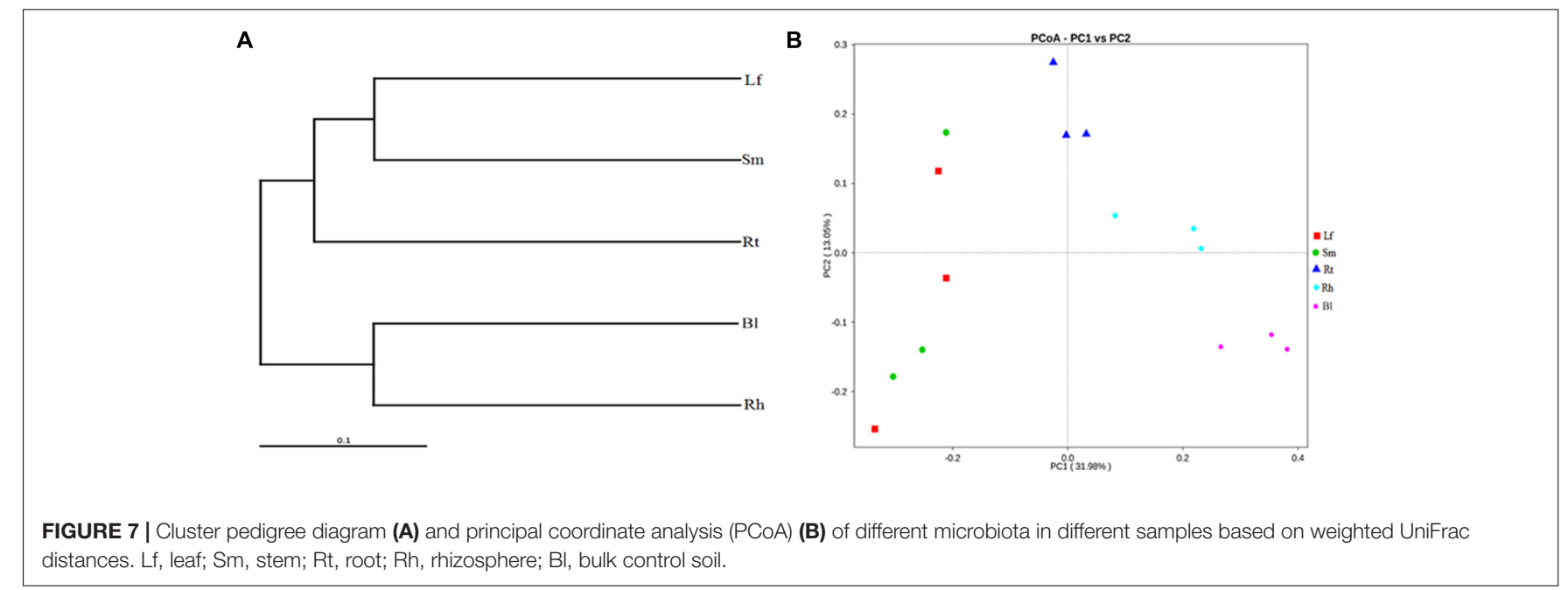

The bacterial communities differed according to the different organs and habitats of $M$. sibirica. To compare the species compositions observed in the $\mathrm{Bl}$ control soil, $\mathrm{Rh}, \mathrm{Rt}, \mathrm{Sm}$, and Lf samples, Adonis was used to determine mean differences and their directionalities (sign) between two samples (Table 2). Based on these tests, a significant difference was observed between the $\mathrm{Bl}$ control soil and Sm samples $\left(R^{2}=0.55\right.$,
$P<0.05)$, and Rt $\left(R^{2}=0.59, P<0.05\right)$, as well as between the Rt and Lf $\left(R^{2}=0.38, P<0.05\right)$, and $\operatorname{Sm}\left(R^{2}=0.5\right.$, $P<0.05)$ samples. No significant difference was observed, however, between the Lf and Sm samples. The results showed that a large number of bacteria inhabited the $\mathrm{Rh}, \mathrm{Bl}$ control soil, and Rt samples compared to that in the Lf and Sm samples. 
TABLE 2 | Adonis analysis of the difference among samples associated with M. sibirica.

\begin{tabular}{llll}
\hline Habitats & Leaf & Stem & Root \\
\hline Bulk control soil & $R^{2}=0.43, P=0.1$ & $R^{2}=0.55, P=0.013$ & $R^{2}=0.59, P=0.0013$ \\
Rhizosphere & $R^{2}=0.36, P=0.1$ & $R^{2}=0.41, P=0.1$ & $R^{2}=0.55, P=0.1$ \\
Root & $R^{2}=0.38, P=0.0013$ & $R^{2}=0.50, P=0.0013$ & \\
Stem & $R^{2}=0.16, P=0.7014$ & & \\
\hline
\end{tabular}

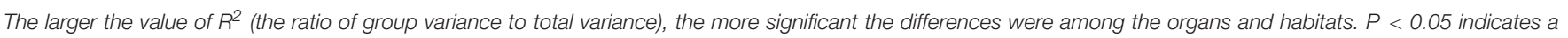
high reliability of the test.

Beta-diversity analysis based on Unweighted Pair Group Method with Arithmetic Mean (UPGMA) clustering (Figure 7A) and PCoA (Figure 7B) were performed to compare the microbial compositions of different samples. Consistent with the results of the Adonis analysis, two different clusters were observed at the genus level in the UPGMA tree: group 1 consisted of the Lf, Sm, and Rt samples, and group 2 consisted of the $\mathrm{Rh}$ and $\mathrm{Bl}$ samples. This indicates that the microbiota of the Lf samples was more similar to those of the Sm and Rt samples than to other samples. Similarly, the Bl and Rh samples exhibited more similar bacterial compositions versus the $\mathrm{Bl}$ and Lf samples. PCoA revealed the main variations in bacterial community composition among the samples. The highest variations in the microbiota of different samples were $31.98 \%$ (PC1) and $13.05 \%$ (PC2), representing a strong separation based on the plant organs or habitats. Samples of the $\mathrm{Rt}, \mathrm{Rh}$, and $\mathrm{Bl}$ control soil from different $M$. sibirica plants clustered together, but the other samples showed some separation.

\section{DISCUSSION}

Based on OTU analysis and the Chaol, ACE, and Shannon's diversity indices, the species diversity and richness were higher in the Rt of $M$. sibirica than in the Sm as well as Lf $(P<0.05$, Table 1). Similar results were also reported for other plants, including Oryza sativa (Mano et al., 2007) and the halophyte Phragmites australis (Ma et al., 2013). Therefore, it could be concluded that the endophytic bacteria distribution varied greatly in different plant tissues, and the Rt hide more bacteria communities than Lf or Sm. Since most endophytic bacteria are from soil origin (Lamb et al., 1996; Hallmann et al., 1999), largest endophytic bacteria diversity in Rt may be attributed to the primary site of interaction between plants and soil (Hardoim et al., 2011). Meanwhile, the lower diversity in Lf may be related to the relatively low diversity of bacteria in the phyllosphere (Lindow and Brandl, 2003), as some endophytic bacteria in Lf may be derived from phyllosphere (Mano et al., 2007).

Previous data have verified that bacterial communities in the $\mathrm{Rh}$ exhibit higher richness than endophytes in the organs of halophytes (Szymańska et al., 2016; Tan et al., 2017). Consistent with these findings, the bacterial species diversity and richness in Rh soil samples of $M$. sibirica were greater than Rt samples. This difference can be explained partly by the environmental variability. Rh are rich in carbon sources secreted by plants, which can be used by bacteria (Wawrik et al., 2005), while Rt represent a relative stable niche for microorganisms. We also found that $\mathrm{Bl}$ control soil samples, which contained $33.8 \%$ of the total number of OTUs, showed higher diversity and richness than the samples associated with $M$. sibirica. Gomes et al. (2014) reported similar results, in which OTU richness was higher in the $\mathrm{Bl}$ soil than in the $\mathrm{Rh}$ of two mangroves, Laguncularia racemosa and Avicennia schaueriana. This effect of reduced bacterial richness in the plant $\mathrm{Rh}$ (compared to the $\mathrm{Bl}$ control soil) has been previously described as the "rhizosphere effect” (Sørensen et al., 1997). However, there are some reports contrary to the above findings. Yang et al. (2017) observed that rhizospheric samples of maize were more diverse than $\mathrm{Bl}$ soil samples. Similar results were also obtained in some halophytes (Fahmy and Ai-Thani, 2008). These different results indicated that the local environment and plant species had complex impacts on bacteria community.

Cluster analysis showed that bacterial communities varied across the different habitats. The $\mathrm{Bl}$ control soil, $\mathrm{Rh}$, Rt, Sm, and Lf of M. sibirica were colonized by the same microbial families, but at different proportions. Proteobacteria, Actinobacteria, Firmicutes, and Bacteroidetes were most abundant phyla for endophytic and $\mathrm{Rh}$ bacteria (Figure 3), in agreement of previous studies about other plants with culture-independent (Jin et al., 2014) and culturedependent methods (Szymańska et al., 2016). However, Bacteroidetes and Proteobacteria mainly appeared in Lf and Rt samples, while Actinobacteria and Firmicutes were dominant in the $\mathrm{Rh}$ and $\mathrm{Bl}$ control soil samples, indicating the presence of habitat specificity (Figure 4). Sequences assigned to Actinobacteria were more abundant in Rhassociated communities than in endophytic communities, which reflects a pattern noted in previous studies (Rastogi et al., 2012; Bodenhausen et al., 2013). In addition, the dominant phyla Actinobacteria and Firmicutes might play important roles in the ecology of $M$. sibirica, as these phyla are also common in other halophytes (Mora-Ruiz et al., 2015; Shi et al., 2015). Therefore, it is not surprising that relatively high abundances of Actinobacteria were found across all organs of $M$. sibirica and in the $\mathrm{Rh}$ and $\mathrm{Bl}$ control soil. Interestingly, Acidobacteria was relatively abundant in $\mathrm{Bl}$ control soil, even though this zone was alkaline, demonstrating that Acidobacteria colonization was not limited to acidic soil. This conclusion was also drawn by Xiong et al. (2012).

Among the top 35 genera, the dominant endophytes belonged to Rhizobium, Streptomyces, and Pseudomonas, many 
species of which have been reported to have beneficial effects for $\mathrm{C}$ or $\mathrm{N}$ cycling in the soil (Herridge, 2013). In addition, Methylobacterium, Sphingomonas, and Actinoplanes were common, and these play a role in plant-microbe interactions in halophytic ecosystems (Omer et al., 2004; Solans et al., 2011; Li et al., 2013). The Methylobacterium genus has been implicated in plant growth and shown effective in promoting seed germination (Omer et al., 2004). This genus also participates in plant $\mathrm{N}$ metabolism since most of these bacteria are capable of $\mathrm{N}$ fixation (Raja et al., 2006). Above all, we speculate that many beneficial microorganisms are present in $M$. sibirica, but the specific interactions between the bacteria and their host require further investigation.

Our results suggest that $M$. sibirica drove differentiation of the bacterial community structure. As verified by Adonis, significant distinctions occurred in the bacterial communities of various samples, with the exception of the Lf and Sm (Table 2). Compared to those in the $\mathrm{Rh}$ and $\mathrm{Bl}$ control soil, endophytic bacteria of the Lf, Sm, and Rt of $M$. sibirica shared more similarity (Figure 7A). It suggested that the endophytic bacteria of Lf, Sm, and Rt samples may share the same origin as mentioned above. The more endophytic bacterial similarity between the Lf and Sm may be related to some strains deriving from phylloplane and caulosphere as revealed in other studies (Mano et al., 2007). Slightly different from our results, Jin et al. (2014) reported that Lf and Sm samples of Stellera chamaejasme were clustered together, but were different from those of $\mathrm{Rh}$ and Rt. As demonstrated by PCoA, habitats explained $31.98 \%$ of the variation in the community structure, while sampling zones explained 13.05\% (Figure 7B). Therefore, data from this study and previous investigations (Ma et al., 2013; Szymańska et al., 2016) indicate that M. sibirica and its tissues have a greater influence than sampling area for bacterial communities.

\section{REFERENCES}

Bais, H. P., Weir, T. L., Perry, L. G., Gilroy, S., and Vivanco, J. M. (2006). The role of root exudates in rhizosphere interactions with plants and other organisms. Annu. Rev. Plant Biol. 57, 233-266. doi: 10.1146/annurev.arplant.57.032905. 105159

Beckers, B., Op De Beeck, M., Thijs, S., Truyens, S., Weyens, N., Boerjan, W., et al. (2016). Performance of $16 \mathrm{~s}$ rDNA primer pairs in the study of rhizosphere and endosphere bacterial microbiomes in metabarcoding studies. Front. Microbiol. 7:650. doi: 10.3389/fmicb.2016. 00650

Bodenhausen, N., Horton, M. W., and Bergelson, J. (2013). Bacterial communities associated with the leaves and the roots of Arabidopsis thaliana. PLOS ONE 8:e56329. doi: 10.1371/journal.pone.0056329

Chen, H. B. (2012). VennDiagram: Generate High-Resolution Venn and Euler Plots. $R$ Package Version 113

Chen, S., Zhang, M., Wang, J., Lv, D., Ma, Y., Zhou, B., et al. (2017). Biocontrol effects of Brevibacillus laterosporus AMCC100017 on potato common scab and its impact on rhizosphere bacterial communities. Biol. Control 106, 89-98. doi: 10.1016/j.biocontrol.2017.01.005

Edgar, R. C., Haas, B. J., Clemente, J. C., Quince, C., and Knight, R. (2011). UCHIME improves sensitivity and speed of chimera detection. Bioinformatics 27, 2194-2200. doi: 10.1093/bioinformatics/ btr381

\section{CONCLUSION}

This study is the first to elucidate the bacterial diversity and composition of the coastal halophyte $M$. sibirica using high-throughput sequencing methods. The dominant bacteria associated with $M$. sibirica were Proteobacteria, Actinobacteria, and Firmicutes. We demonstrated that the bacterial communities varied across the different habitats of Lf, Sm, Rt, the Rh, and $\mathrm{Bl}$ control soil. Our results showed that $M$. sibirica affected bacterial diversity and structured the bacterial community. These results provide insight into the complex microbial compositions of coastal halophytes. Further studies are necessary to elucidate the functional roles of these bacterial species in plant-microbe interactions in the inter-tidal zone, for example, how they may enhance or impair plant fitness.

\section{AUTHOR CONTRIBUTIONS}

C-SZ contributed to the conception of the study and wrote the manuscript. X-YT performed the experiments and data analyses.

\section{ACKNOWLEDGMENT}

This work was supported by the Agricultural Science and Technology Innovation Program of China (grant no. ASTIPTRIC07).

\section{SUPPLEMENTARY MATERIAL}

The Supplementary Material for this article can be found online at: https://www.frontiersin.org/articles/10.3389/fmicb. 2017.02288/full\#supplementary-material

Etesami, H., Alikhani, H. A., and Hosseini, H. M. (2015). Indole-3-acetic acid (IAA) production trait, a useful screening to select endophytic and rhizosphere competent bacteria for rice growth promoting agents. MethodsX 2, 72-78. doi: 10.1016/j.mex.2015.02.008

Fahmy, G. M., and Ai-Thani, R. F. (2008). Diversity of halophytes and soil bacteria in the coastal salt marsh of Al-Dhakhira, Qatar. A Paper Presented before the First International Congress of "Documenting, Analysing and Managing Biodiversity in the Middle East, Aqaba.

Gomes, N. C. M., Cleary, D. F. R., Pires, A. C. C., Almeida, A., Cunha, A., Mendonça-Hagler, L. C. S., et al. (2014). Assessing variation in bacterial composition between the rhizospheres of two mangrove tree species. Estuar. Coast. Shelf Sci. 139, 40-45. doi: 10.1016/j.ecss.2013.12.022

Hallmann, J., Rodriguezkabana, R., and Kloepper, J. W. (1999). Chitin-mediated changes in bacterial communities of the soil, rhizosphere and within roots of cotton in relation to nematode control. Soil Biol. Biochem. 31, 551-560. doi: 10.1016/S0038-0717(98)00146-1

Haque, M. A., Jin, H. L., and Cho, K. M. (2015). Endophytic bacterial diversity in Korean kimchi made of Chinese cabbage leaves and their antimicrobial activity against pathogens. Food Control 56, 24-33. doi: 10.1016/j.foodcont.2015. 03.006

Hardoim, P. R., Andreote, F. D., Reinhold-Hurek, B., Sessitsch, A., van Overbeek, L. S., and van Elsas, J. D. (2011). Rice root-associated bacteria: insights into community structures across 10 cultivars. FEMS Microbiol. Ecol. 77, 154-164. doi: 10.1111/j.1574-6941.2011.01092.x 
Hashem, A., Abd_Allah, E. F., Alqarawl, A. A., Al-Huqall, A. A., Wirth, S., and Egamberdieva, D. (2016). The interaction between arbuscular mycorrhizal fungi and endophytic bacteria enhances plant growth of Acacia gerrardii under salt stress. Front. Microbiol. 7:868. doi: 10.3389/fmicb.2016.01089

Herridge, D. (2013). Rhizobial Inoculants Fact Sheet. Grains Research and Development Corporation. Available at: https://grdc.com.au/cm/link.aspx/7e/ media/rhizobial-inoculants-fact-sheet.pdf

Jin, H., Yang, X. Y., Yan, Z. Q., Liu, Q., Li, X. Z., Chen, J. X., et al. (2014). Characterization of rhizosphere and endophytic bacterial communities from leaves, stems and roots of medicinal Stellera chamaejasme L. Syst. Appl. Microbiol. 37, 376-385. doi: 10.1016/j.syapm.2014.05.001

Jin, W., Zhou, R., Zhao, H., and Zhao, Y. (2012). Growth and physiological adaptation of Messerschmidia sibirica to sand burial on coastal sandy. Acta Ecol. Sin. 32, 4291-4299. doi: 10.5846/stxb201106270948

Kolde, R. (2012). pheatmap: Pretty Heatmaps. R Package Version 061.

Kuczynski, J., Stombaugh, J., Walters, W. A., González, A., Caporaso, J. G., and Knight, R. (2011). Using QIIME to analyze 16S rRNA gene sequences from microbial communities. Curr. Protoc. Bioinformatics 36, 10.7.1-10.7.20. doi: 10.1002/0471250953.bi1007s36

Laanbroek, H. J., Keijzer, R. M., Verhoeven, J. T. A., and Whigham, D. F. (2013). Changes in community composition of ammonia-oxidizing betaproteobacteria from stands of black mangrove (Avicennia germinans) in response to ammonia enrichment and more oxic conditions. Front. Microbiol. 4:343. doi: 10.3389/ fmicb.2013.00343

Lamb, T. G., Tonkyn, D. W., and Kluepfel, D. A. (1996). Movement of Pseudomonas aureofaciens from the rhizosphere to aerial plant tissue. Can. J. Microbiol. 42, 1112-1120. doi: 10.1139/m96-143

Li, Y., Chen, Q., Wang, C. H., Shu, C., Jian, H., Xing, H., et al. (2013). Degradation of acetochlor by consortium of two bacterial strains and cloning of a novel amidase gene involved in acetochlor-degrading pathway. Bioresour. Technol. 148, 628-631. doi: 10.1016/j.biortech.2013.09.038

Lindow, S. E., and Brandl, M. T. (2003). Microbiology of the phyllosphere. Appl. Environ. Microbiol. 69, 1875-1883. doi: 10.1128/AEM.69.4.1875-1883.2003

Ma, B., Lv, X., Warren, A., and Gong, J. (2013). Shifts in diversity and community structure of endophytic bacteria and archaea across root, stem and leaf tissues in the common reed, Phragmites australis, along a salinity gradient in a marine tidal wetland of northern China. Antonie Van Leeuwenhoek 104, 759-768. doi: 10.1007/s10482-013-9984-3

Magoč, T., and Salzberg, S. L. (2011). FLASH: fast length adjustment of short reads to improve genome assemblies. Bioinformatics 27, 2957-2963. doi: 10.1093/ bioinformatics/btr507

Majeed, A., Abbasi, M. K., Hameed, S., Imran, A., and Rahim, N. (2015). Isolation and characterization of plant growth-promoting rhizobacteria from wheat rhizosphere and their effect on plant growth promotion. Front. Microbiol. 6:198. doi: $10.3389 /$ fmicb. 2015.00198

Mano, H., Tanaka, F., Nakamura, C., Kaga, H., and Morisaki, H. (2007). Culturable endophytic bacterial flora of the maturing leaves and roots of rice plants (Oryza sativa) cultivated in a paddy field. Microbes Environ. 22, 175-185. doi: 10.1264/ jsme2.22.175

Mora-Ruiz, M. R., Font-Verdera, F., Díaz-Gil, C., Urdiain, M., RodríguezValdecantos, G., González, B., et al. (2015). Moderate halophilic bacteria colonizing the phylloplane of halophytes of the subfamily Salicornioideae (Amaranthaceae). Syst. Appl. Microbiol. 38, 406-416. doi: 10.1016/j.syapm.2015. 05.004

Navarro-Torre, S., Barcia-Piedras, J. M., Mateos-Naranjo, E., Redondo-Gómez, S., Camacho, M., Caviedes, M. A., et al. (2017). Assessing the role of endophytic bacteria in the halophyte Arthrocnemum macrostachyum salt tolerance. Plant Biol. 19, 249-256. doi: 10.1111/plb.12521

Nenadic, O., and Greenacre, M. (2007). Correspondence analysis in R, with twoand three-dimensional graphics: the ca package. J. Stat. Softw. 20, 1704-1710.

Oksanen, J. F., Blanchet, F. G., Kindt, R., Legendre, P., O’Hara, R. B., Simpson, G. L., et al. (2011). Vegan: Community Ecology Package. R Package Version $1.17-8$.

Omer, Z. S., Tombolini, R., Broberg, A., and Gerhardson, B. (2004). Indole-3-acetic acid production by pink-pigmented facultative methylotrophic bacteria. Plant Growth Regul. 43, 93-96. doi: 10.1023/B:GROW.0000038360.09079.ad

Pereira, S. I. A., and Castro, P. M. L. (2014). Diversity and characterization of culturable bacterial endophytes from Zea mays and their potential as plant growth-promoting agents in metal-degraded soils. Environ. Sci. Pollut. Res. 21, 14110-14123. doi: 10.1007/s11356-014-3309-6

Raja, P., Uma, S., and Sundaram, S. (2006). Non-nodulating pink-pigmented facultative Methylobacterium sp. with a functional nifH gene. World J. Microbiol. Biotechnol. 22, 1381-1384. doi: 10.1007/s11274-0069199-0

Ramírez-Elías, M. A., Ferrera-Cerrato, R., Alarcón, A., Almaraz, J. J., RamírezValverde, G., De-Bashan, L. E., et al. (2014). Identification of culturable microbial functional groups isolated from the rhizosphere of four species of mangroves and their biotechnological potential. Appl. Soil Ecol. 82, 1-10. doi: 10.1016/j.apsoil.2014.05.001

Rastogi, G., Sbodio, A., Tech, J. J., Suslow, T. V., Coaker, G. L., and Leveau, J. H. J. (2012). Leaf microbiota in an agroecosystem: spatiotemporal variation in bacterial community composition on field-grown lettuce. ISME J. 6, 1812-1822. doi: 10.1038/ismej.2012.32

Segata, N., Izard, J., Waldron, L., Gevers, D., Miropolsky, L., Garrett, W. S., et al. (2011). Metagenomic biomarker discovery and explanation. Genome Biol. 12:R60. doi: 10.1186/gb-2011-12-6-r60

Shahzad, R., Khan, A. L., Bilal, S., Waqas, M., Kang, S. M., and Lee, I. J. (2017). Inoculation of abscisic acid-producing endophytic bacteria enhances salinity stress tolerance in Oryza sativa. Environ. Exp. Bot. 136, 68-77. doi: 10.1016/j. envexpbot.2017.01.010

Shi, Y. W., Lou, K., Li, C., Wang, L., Zhao, Z. Y., Zhao, S., et al. (2015). Illuminabased analysis of bacterial diversity related to halophytes Salicornia europaea and Sueada aralocaspica. J. Microbiol. 53, 678-685. doi: 10.1007/s12275-0155080- $\mathrm{X}$

Shin, D., Park, M. S., Jung, S., Lee, M. S., Lee, K. H., Bae, K. S., et al. (2007). Plant growth-promoting potential of endophytic bacteria isolated from roots of coastal sand dune plants. J. Microbiol. Biotechnol. 17, 1361-1368.

Silveira, A. P. D. D., Sala, V. M. R., and Labanca, E. G. (2016). Nitrogen metabolism and growth of wheat plant under diazotrophic endophytic bacteria inoculation. Appl. Soil Ecol. 107, 313-319. doi: 10.1016/j.apsoil.2016.07.005

Smalla, K., Wieland, G., Buchner, A., Zock, A., Parzy, J., Kaiser, S., et al. (2001). Bulk and rhizosphere soil bacterial communities studied by denaturing gradient gel electrophoresis: plant-dependent enrichment and seasonal shifts revealed. Appl. Environ. Microbiol. 67, 4742-4751. doi: 10.1128/AEM.67.10.4742-4751.2001

Solans, M., Vobis, G., Cassa, F., Luna, V., and Wall, L. G. (2011). Production of phytohormones by root-associated saprophytic actinomycetes isolated from the actinorhizal plant Ochetophila trinervis. World J. Microbiol. Biotechnol. 27, 2195-2202. doi: 10.1007/s11274-011-0685-7

Sørensen, J., Elsas, J. D. V., Trevors, J. T., and Wellington, E. M. H. (1997). “The rhizosphere as a habitat for soil microorganisms," in Modern Soil Microbiology, eds J. D. V. Elsas, J. T. Trevors, and E. M. H. Wellington (New York, NY: Maccel Dekker), 21-45.

Syranidou, E., Christofilopoulos, S., Gkavrou, G., Thijs, S., Weyens, N., Vangronsveld, J., et al. (2016). Exploitation of endophytic bacteria to enhance the phytoremediation potential of the wetland helophyte Juncus acutus. Front. Microbiol. 7:1016. doi: 10.3389/fmicb.2016.01016

Szymañska, S., Płociniczak, T., Piotrowskaseget, Z., and Hrynkiewicz, K. (2016). Endophytic and rhizosphere bacteria associated with the roots of the halophyte Salicornia europaea L. - community structure and metabolic potential. Microbiol. Res. 192, 37-51. doi: 10.1016/j.micres.2016.05.012

Tan, Y., Cui, Y., Li, H., Kuang, A., Li, X., Wei, Y., et al. (2017). Diversity and composition of rhizospheric soil and root endogenous bacteria in Panax notoginseng during continuous cropping practices. J. Basic Microbiol. 23, 714-716.

Wang, D., and Zheng, Z. (2016). Research progress of Messerschmidia sibirica Linn. Chin. Agric. Sci. Bull. 32, 21-24.

Wang, Q., Garrity, G. M., Tiedje, J. M., and Cole, J. R. (2007). Naive Bayesian classifier for rapid assignment of rRNA sequences into the new bacterial taxonomy. Appl. Environ. Microbiol. 16, 5261-5267. doi: 10.1128/AEM. 00062-07

Wawrik, B., Kerkhof, L., Kukor, J., and Zylstra, G. (2005). Effect of different carbon sources on community composition of bacterial enrichments from soil. Appl. Environ. Microbiol. 71, 6776-6783. doi: 10.1128/AEM.71.11.6776-6783. 2005

Wiegert, R. G., Pomeroy, L. R., and Wiebe, W. J. (1981). Ecology of Salt Marshes: An Introduction. New York, NY: Springer. 
Xiang, X. L., Chu, Q. G., Liu, Z. Q., and Qin, Y. S. (2008). Correlation between the morphological structure of salt gland and salt secretion in Messerschmidia sibirica Linn. J. Jinan Univ. Nat. Sci. Med. Ed. 29, 305-310.

Xiong, J. B., Liu, Y. Q., Lin, X. G., Zhang, H. Y., Zeng, J., Hou, J. Z., et al. (2012). Geographic distance and $\mathrm{pH}$ drive bacterial distribution in alkaline lake sediments across Tibetan Plateau. Environ. Microbiol. 14, 2457-2466. doi: 10.1111/j.1462-2920.2012.02799.x

Xu, L., Zhang, Y., Wang, L., Chen, W., and Wei, G. (2014). Diversity of endophytic bacteria associated with nodules of two indigenous legumes at different altitudes of the Qilian Mountains in China. Syst. Appl. Microbiol. 37, 457-465. doi: 10.1016/j.syapm.2014.05.009

Yang, Y., Wang, N., Guo, X., Zhang, Y., and Ye, B. (2017). Comparative analysis of bacterial community structure in the rhizosphere of maize by high-throughput pyrosequencing. PLOS ONE 12:e0178425. doi: 10.1371/journal.pone. 0178425

Yi, H., Ahn, Y., Song, G. C., Ghim, S., Lee, S., Lee, G., et al. (2016). Impact of a bacterial volatile 2, 3-butanediol on Bacillus subtilis rhizosphere robustness. Front. Microbiol. 7:993. doi: 10.3389/fmicb.2016. 00993
Zhang, C., and Kong, F. (2014). Isolation and identification of potassiumsolubilizing bacteria from tobacco rhizospheric soil and their effect on tobacco plants. Appl. Soil Ecol. 82, 18-25. doi: 10.1016/j.apsoil.2014. 05.002

Zhao, S., Zhou, N., Zhao, Z., Zhang, K., Wu, G., and Tian, C. (2016). Isolation of endophytic plant growth-promoting bacteria associated with the halophyte Salicornia europaea and evaluation of their promoting activity under salt stress. Curr. Microbiol. 73, 574-581. doi: 10.1007/s00284-0161096-7

Conflict of Interest Statement: The authors declare that the research was conducted in the absence of any commercial or financial relationships that could be construed as a potential conflict of interest.

Copyright (C) 2017 Tian and Zhang. This is an open-access article distributed under the terms of the Creative Commons Attribution License (CC BY). The use, distribution or reproduction in other forums is permitted, provided the original author(s) or licensor are credited and that the original publication in this journal is cited, in accordance with accepted academic practice. No use, distribution or reproduction is permitted which does not comply with these terms. 